Pacific Journal of Mathematics

COMPARISON OF THE STATES OF CLOSED LINEAR 


\title{
COMPARISON OF THE STATES OF CLOSED LINEAR TRANSFORMATIONS
}

\author{
J. D. FAIRES
}

Let $X$ and $Y$ be Banach spaces and $T$, respectively $S$, be a bounded linear transformation mapping $X$ into $Y$, respectively $Y$ into $X$. It is well-known that a nonzero complex number $\lambda$ belongs to the spectrum of $S T$ precisely when $\lambda$ belongs to the spectrum of $T S$. The main result of $\S 2$ shows that for $\lambda \neq 0$ the states of the operators $S T-\lambda I_{X}, T S-\lambda I_{Y}$ agree.

Sufficient conditions are obtained for this same result to hold when $T$ and $S$ are unbounded closed linear transformations from $X$ into $Y$ and $Y$ into $X$ respectively. Section 4 compares spectral decompositions of $S T$ and $T S$ when these sufficient conditions are satisfied.

Throughout this paper $D(A)$ and $R(A)$ will denote the domain and range of $A$. The resolvent of $A$ will be denoted $\rho(A)$, the spectrum $\sigma(A)$, the point spectrum $p(A)$ and the approximate point spectrum $a(A)$. [X, $Y]$ will denote the set of all bounded linear transformations, defined on the Banach space $X$ into the Banach space $Y$. Any other notation used will agree with that of [3]. When no confusion will arise the identity operator will be denoted by $I$ regardless of the space. The following preliminary result can be easily varified.

Proposition 1.1. If $T: D(T) \subset X \rightarrow Y, S: D(S) \subset Y \rightarrow X$ and $\lambda \neq 0$, then $\lambda \in p(T S)$ if and only if $\lambda \in p(S T)$.

\section{Continuous transformations.}

Proposition 2.1. If $\lambda \neq 0$ then $\overline{R(S T-\lambda I)}=X$ precisely when $\overline{R(T S-\lambda I)}=Y$.

Proof. $\overline{R(S T-\lambda I)} \neq X$ implies that there exists an $x^{\prime} \in X^{\prime}$, $x^{\prime} \neq 0$ such that $x^{\prime}((S T-\lambda I)(x))=0$ for all $x \in X$. Consequently for all $x \in X, 0=(S T-\lambda I)^{\prime}\left(x^{\prime}(x)\right)=\left(T^{\prime} S^{\prime}-\lambda I\right)\left(x^{\prime}(x)\right)$ and $\lambda \in p\left(T^{\prime} S^{\prime}\right)$. By Proposition 1.1, $\lambda \in p\left(S^{\prime} T^{\prime}\right)$ so $y^{\prime} \in Y^{\prime}, y^{\prime} \neq 0$ exists with the property that for each $y \in Y, 0=\left(S^{\prime} T^{\prime}-\lambda I\right)\left(y^{\prime}(y)\right)=y^{\prime}((T S-\lambda I)(y))$. Thus $\overline{R(T S-\lambda I)} \neq Y$.

The following is a construction of a "generalized" Banach space in the manner of that of Berberian [2].

Denote by glim a fixed "generalized Banach limit" defined for all 
bounded sequences of complex numbers and having properties: ([1] page 34)

(i) $\operatorname{glim}\left(\lambda_{n}+\mu_{n}\right)=\operatorname{glim} \lambda_{n}+\operatorname{glim} \mu_{n}$;

(ii) $\operatorname{glim}\left(\lambda \lambda_{n}\right)=\lambda \operatorname{glim} \lambda_{n}$;

(iii) $\operatorname{glim} \lambda_{n}=\lim \lambda_{n}$ if $\left\{\lambda_{n}\right\}$ converges;

(iv) $\operatorname{glim} \lambda_{n} \geqq 0$ whenever $\lambda_{n} \geqq 0$ for each $n$.

For a Banach space $X$, denote by $\mathscr{B}(X)$ the set of all sequences $\left\{x_{n}\right\}$ of elements of $X$ for which sup $\left\|x_{n}\right\|<\infty$. If for $s=\left\{x_{n}\right\}$ and $t=\left\{y_{n}\right\}$ in $\mathscr{B}(X)$ and complex $\lambda$ we define $s+t=\left\{x_{n}+y_{n}\right\}, \lambda s=\left\{\lambda x_{n}\right\}$ and $\|s\|_{1}=\operatorname{glim}\left\|x_{n}\right\|$ it is clear that $\mathscr{B}(X)$ is a prenormed space. If $\mathscr{N}(X)=\left\{s \in \mathscr{B}(X):\|s\|_{1}=0\right\}$ then $\mathscr{P}(X)=\mathscr{B}(X) / \mathscr{N}(X)$ is a normed vector space whose completion will be denoted by $\mathscr{K}(X)$. Since $x \mapsto\{x\}+\mathscr{N}(X)$ is an isomorphism of $X$ into a closed linear subspace $X^{\prime}$ of the Banach space $\mathscr{K}(X), X$ can be identified with this subspace and $X^{\prime}$ is called the generalized extension of $X$.

For $T \in[X, Y]$ define $\mathscr{B}(T): \mathscr{B}(X) \rightarrow \mathscr{B}(Y)$ as $\mathscr{B}(T): s=\left\{x_{n}\right\} \mapsto$ $\left\{T x_{n}\right\} . \quad T$ is bounded so $\mathscr{B}(T)$ is bounded and $\|\mathscr{B}(T)\|_{1}=\|T\|$. Moreover, $\mathscr{B}(T): \mathscr{N}(X) \rightarrow \mathscr{N}(Y)$ so $\mathscr{B}(T)$ may be extended to $\mathscr{P}(X)$ and consequently to $X^{\prime}$ to obtain a unique extension $T^{\prime} \in\left[X^{\prime}, Y^{\prime}\right]$ of $T$ with $\left\|T^{\prime}\right\|=\|T\|$.

For $T_{1}, T_{2} \in[X, Y]$ and $S \in[Y, X]$ the following properties can be verified directly:

(i ) $\left(T_{1}+T_{2}\right)^{\prime}=T_{1}^{\prime}+T_{2}^{\prime}$;

(ii) $\left(\lambda T_{1}\right)^{\prime}=\lambda T_{1}^{\prime}$;

(iii) $\left(S T_{1}\right)^{\prime}=S^{\prime} T_{1}^{\prime}$.

The next proposition gives the results which necessitated the preceding construction. For the Hilbert space analogue of this proposition, see Berberian [2], Theorem 1.

\section{Proposition 2.2. Let $A \in[X, X]$ then $a(A)=a\left(A^{\prime}\right)=p(A)$.}

Proof. $\lambda \in a\left(A^{\prime}\right)$ implies that for each $\varepsilon>0$ an $s \in X^{\prime}$ exists with $\left\|\left(A^{\prime}-\lambda I\right) s\right\|<\varepsilon\|s\|$. Since $\mathscr{P}(x)$ is dense in $X^{\prime}$, it may be assumed that $s=\left\{x_{n}\right\} \in \mathscr{P}(X)$. Thus $\left\|\left(A^{\prime}-\lambda I\right) s\right\|=\operatorname{glim}\left\|(A-\lambda I) x_{n}\right\|<$ $\varepsilon \operatorname{glim}\left\|x_{n}\right\|$ so $0>\operatorname{glim}\left[\varepsilon\left\|x_{n}\right\|-\left\|(A-\lambda I) x_{n}\right\|\right]$. By property (iv) of glim it must be true that for at least one $n, 0<\varepsilon\left\|x_{n}\right\|-\left\|(A-\lambda I) x_{n}\right\|$ and hence for some $x_{n} \in X$, $\left\|(A-\lambda I) x_{n}\right\|<\varepsilon\left\|x_{n}\right\|$, which implies $\lambda \in a(A)$.

To complete the proof of this proposition, if suffices to show that $a(A) \subset p\left(A^{\prime}\right)$. For $\lambda \in a(A)$, a sequence $\left\{x_{n}\right\}$ in $X$ exists with $\left\|x_{n}\right\|=1$ for all $n$ and $\left\|(A-\lambda I) x_{n}\right\| \rightarrow 0$. $\left\{x_{n}\right\}$ is bounded in norm so $s=\left\{x_{n}\right\} \in X^{\prime}$, $\|s\|=1$ and $\left\|\left(A^{\prime}-\lambda I\right) s\right\|=\operatorname{glim}\left\|(A-\lambda I) x_{n}\right\|=0$. Hence $\lambda \in p\left(A^{\prime}\right)$.

Considering $T \in[X, Y]$ and $S \in[Y, X]$ we obtain: 
Corollary 2.1. If $\lambda \neq 0$ then $\lambda \in a(T S)$ if and only if $\lambda \in a(S T)$.

Proof. By Proposition 2.2, $\lambda \in a(T S)$ implies $\lambda \in p\left((T S)^{\prime}\right)=p\left(T^{\prime} S^{\prime}\right)$. Hence by Proposition 1.1, $\lambda \in p\left(S^{\prime} T^{\prime}\right)=a(S T)$.

The preceding corollary together with the result of Propositions 1.1 and 2.1 prove the following theorem. The classification of states of a linear operator may be found in [5].

TheOREM 2.1. If $T \in[X, Y], S \in[Y, X]$ and $\lambda \neq 0$, then the states of $T S-\lambda I$ and $S T-\lambda I$ agree.

Proof. To show that one of the operators cannot be in state $I_{3}$ while the other is in state $I I_{3}$, a theorem of Goldberg [4], Theorem II 4.4, is used which in our case states:

(i) $T$ has a bounded inverse if and only if $R\left(T^{*}\right)=X^{*}$.

(ii) $T^{*}$ has a bounded inverse if and only if $R(T)=Y$.

3. Closed transformations. Let $T$ be a closed linear transformation with $D(T)$ and $R(T)$ both contained in the Banach space $X$. Suppose further that $\rho(T) \neq \phi$, that $\alpha \in \rho(T)$ is fixed and $A \in[X, X]$ is defined by $A=(T-\alpha I)^{-1}$. The following theorems are due to Taylor [6].

THEOREM 3.1. Suppose "l and $\lambda$ are complex numbers satisfying $(\lambda-\alpha) \mu=1$ :

(i) If $x \in X$ and $(\mu I-A) x=y$ then $(T-\lambda I)(\mu x-y)=\mu^{-1} y$;

(ii) If $x \in D(T)$ and $(T-\lambda I) x=y$ then $(\mu I-A) x=\mu A y$. Furthermore, $\mu I-A$ is 1-1 precisely when $T-\lambda I$ is 1-1 and on the common domain of their inverses $(\mu I-A)^{-1}=\mu^{-2}\left[\mu I+(T-\lambda I)^{-1}\right]$ and $(T-\lambda I)^{-1}=\mu(\mu I-A)^{-1} A=\mu A(\mu I-A)^{-1}$.

THEOREM 3.2. Let $\lambda$ and $\mu$ satisfy $(\lambda-\alpha) \mu=1$. Then $\lambda$ belongs to $\rho(T)$ if and only if $\mu$ belongs to $\rho(A)$.

The following lemma follows from the closed graph theorem and will be needed often in our development:

LeMma 3.1. If $P: D(P) \subset Y \rightarrow Z$ is a closed linear transformation and $Q \in[X, Y]$ where $X, Y, Z$ are Banach spaces, then $P Q$ is closed and if $R(Q) \subset D(P)$ then $P Q \in[X, Z]$.

For $T$ closed with $D(T)$ and $R(T)$ both in $X$ and $0 \neq \alpha \in \rho(T)$ we define $A=(T-\alpha I)^{-1}$ and $B=T(T-\alpha I)^{-1}\left(T-\alpha I^{-1}\right)$. (By Lemma 3.1, $B \in[X, X]$.) 
The next three propositions give the substance for a method of referring a pair of closed operators to a pair which are continuous and everywhere defined.

Proposition 3.1. Consider T, $A$, and $B$ as defined above and $0 \neq$ $\alpha \in \rho(T)$. For $0 \neq \lambda \neq \alpha$, let $\nu=\left(\lambda /(\lambda-\alpha)^{2}\right), \mu=(1 /(\lambda-\alpha))$. Then $R(B-\nu I) \subset R(T-\lambda I)$.

Proof. Suppose $y=(B-\nu I) x$. Then $y+\nu x=(T-\alpha I)^{-1}[x+$ $\left.\alpha(T-\alpha I)^{-1} x\right] \in D(T-\alpha I)=D(T-\lambda I)$ and $(T-\lambda I)(y+\nu x)+(\lambda-$ $\alpha)(y+\nu x)=x+\alpha(T-\alpha I)^{-1} x$; so $-1 / \alpha[(T-\lambda I)(y+\nu x)+(\lambda-\alpha) y]=$ $\mu x-A x$. If Theorem 3.1, part (i), is applied, we obtain that $1 / \alpha[(T-\lambda I)(y+\nu x)+(\lambda-\alpha) y] \in R(T-\lambda I)$ so that

$$
\begin{aligned}
& (T-\lambda I)\left\{\mu x+\frac{1}{\alpha}[(T-\lambda I)(y+\nu x)+(\lambda-\alpha) y]\right. \\
& \left.+\frac{1}{\mu \alpha}(y+\nu x)\right\}=\frac{-1}{\alpha \nu \mu} y \in R(T-\lambda I) .
\end{aligned}
$$

Proposition 3.2. If $\lambda \neq 0$ is such that for some $0 \neq \alpha \in \rho(T)$, $\alpha^{2} / \lambda \in \rho(T)$ also, then $R(T-\lambda I) \subset R(B-\nu I)$.

Proof. We may assume, without loss of generality, that $\lambda \neq \alpha$, for if $0 \neq \alpha \in \rho(T)$ there exists some $a>0$ with $0 \notin\{\mu|| \mu-\lambda \mid<a\} \subset$ $\rho(T)$ and $\lambda=\alpha+(a / 2) e^{i \theta}$, where $\theta$ is the argument of $\alpha$, will satisfy our hypothesis.

For $x \in D(T), B T x=x+\alpha A x+\alpha B x$. Consequently, if (T$\lambda I) x=y$, then $(y-\alpha) B x=x+\alpha A x-B y$. Theorem 3.1, part (ii), implies $(\mu I-A) x=\mu A y$ so $(\lambda-\alpha) B x=\lambda \mu x-\alpha \mu A y-B y$. Thus

$$
\begin{aligned}
(B-\nu I)(x+\mu y) & =-\frac{\alpha \nu}{\lambda} A y-\nu \mu y \\
& =-\frac{\alpha \nu}{\lambda}\left[A-\frac{1}{\left(\alpha^{2} / \lambda-\alpha\right)} I\right] y .
\end{aligned}
$$

By hypothesis, $\alpha^{2} / \lambda \in \rho(T)$, so Theorem 3.2 may be used to obtain

$$
\frac{1}{\left(\alpha^{2} / \lambda-\alpha\right)} \in \rho(A)
$$

and

$$
-\frac{\alpha \nu}{\lambda} y=\left[A-\frac{\lambda}{\left(\alpha^{2}-\alpha \lambda\right)} I\right]^{-1}(B-\nu I)(x+\mu y) .
$$

Since $\left[A-\frac{\lambda}{\left(\alpha^{2}-\alpha \lambda\right)} I\right]^{-1}$ and $B-\nu I$ commute, 
(2) $-\frac{\alpha \nu}{\lambda} y=(B-\nu I)\left[A-\frac{1}{\left(\alpha^{2} / \lambda-\alpha\right)} I\right]^{-1}(x+\mu y) \in R(B-\nu I)$.

The following proposition follows easily by considering equations (1) and (2), together with the result of Theorem 3.2.

Proposition 3.3. Suppose $\lambda \neq 0$ and for some $\alpha \neq 0$ in $\rho(T)$, $\alpha^{2} / \lambda \in \rho(T)$ also. Then $T-\lambda I$ is $1-1$ precisely when $B-\nu I$ is $1-1$.

The following theorem is an immediate consequence of the Propositions 3.1, 3.2, 3.3, and the closed graph theorem.

THEOREm 3.3. Let $T$ be a closed linear operator with $D(T)$ and $R(T)$ both contained in the Banach space $X$. Suppose $\lambda \neq 0$ is a complex number with the property that for some $\alpha \in \rho(T), \alpha^{2} / \lambda \in \rho(T)$ also, then the state of $T-\lambda I$ is the same as the state of $B-\nu I$.

For the remainder of this section, we consider a pair of closed linear transformations, $T: D(T) \subset X \rightarrow Y$ and $S: D(S) \subset Y \rightarrow X$, with the property that $S T$ and $T S$ are both closed on their respective domains. We assume moreover that $\rho(T S) \cap \rho(S T) \neq \varnothing$ and for $\alpha \in \rho(T S) \cap \rho(S T)$ fixed we define:

$$
A(S T)=(S T-\alpha I)^{-1}, \quad A(T S)=(T S-\alpha I)^{-1}
$$

and

$$
\begin{aligned}
& B(S T)=S T(S T-\alpha I)^{-1}(S T-\alpha I)^{-1} \\
& B(T S)=T S(T S-\alpha I)^{-1}(T S-\alpha I)^{-1}
\end{aligned}
$$

When $x \in D(T), T A(S T) x=A(T S) T x$; thus $B(S T)$ and $B(T S)$ may be rewritten:

$$
\begin{aligned}
& B(S T)=S(T S-\alpha I)^{-1} T(S T-\alpha I)^{-1}=S A(T S) T A(S T) \\
& B(T S)=T(S T-\alpha I)^{-1} S(T S-\alpha I)^{-1}=T A(S T) S A(T S) .
\end{aligned}
$$

Since $R(A(S T)) \subset D(T)$ and $R(A(T S)) \subset D(S)$, Lemma 3.1 shows that $T A(S T) \in[X, Y]$ and $S A(T S) \in[Y, X]$. By Theorem 2.1, whenever $\nu \neq 0$, the state of $B(T S)-\nu I$ is the same as the state of $B(S T)-\nu I$, which gives the main result in this section:

THEOREM 3.4. If $\lambda \neq 0$ is such that for some $\alpha \in \rho(S T) \cap \rho(T S)$, $\alpha^{2} / \lambda \in \rho(S T) \cap \rho(T S)$ also, the state of $S T-\lambda I$ is the same as the state of $T S-\lambda I$.

It is conjectured that the hypothesis of Theorem 3.4 can be 
weakened to simply requiring that $\rho(S T) \cap \rho(T S) \neq\{0\}$. A different method of proof would likely be needed, however.

In the remainder of this section we consider conditions on the transformations $S$ and $T$ which will ensure that the hypotheses of Theorem 3.4 are fulfilled. We first need the following propositions:

Proposition 3.4. If T, S, TS, and $S T$ are closed and $\lambda \neq 0$ is such that $\lambda \in \sigma(T S) \cap \rho(S T)$, then whenever $\alpha \in \rho(T S) \cap \rho(S T)$,

$$
\frac{\alpha^{2}}{\lambda} \in \sigma(S T)
$$

Proof. Since $\lambda \in \rho(S T), S T-\lambda I$ is $1-1$; so by Theorem 3.4, TS $-\lambda I$ is also $1-1$, and $\lambda \in \sigma(T S)$ implies $\overline{R(T S-\lambda I)} \neq Y$. For $0 \neq \alpha \in \rho(T S)$ and $\nu=\lambda /(\lambda-\alpha)^{2}$ we have by Proposition 3.1

$$
R(B(T S)-\nu I) \subset R(T S-\lambda I)
$$

and consequently

$$
\overline{R(B(T S)-\nu I)} \neq Y .
$$

By Theorem $3.3 R(B(S T)-\nu I) \neq X$. If $\alpha^{2} / \lambda \in \rho(S T)$, then

$$
R(S T-\lambda I) \subset R(B(S T)-\nu I),
$$

so

$$
\overline{R(S T)-\lambda I)} \neq X
$$

This clearly contradicts our assumption of $\lambda \in \rho(S T)$.

Proposition 3.5. If $T, S, T S$, and $S T$ are closed and $\rho_{1}$, respectively $\rho_{2}$, are connected components of $\rho(S T)$, respectively $\rho(T S)$, then $\left(\rho_{1}-\rho_{2}\right) \cup\left(\rho_{2}-\rho_{1}\right) \subset\{0\}$.

Proof. It suffices to show that both $\rho_{1} \cap \partial \rho_{2} \subset\{0\}$, where $\partial \rho_{2}$ denotes the boundary of $\rho_{2}$, and $\rho_{2} \cap \partial \rho_{1} \subset\{0\}$.

To prove the forme $x$, suppose $0 \neq \lambda \in \rho_{1} \cap \partial \rho_{2}$. Then $\lambda \in \sigma(T S)$ and there is an open set $N$ with $\lambda \in N \subset \rho_{1}$. We may therefore construct a sequence $\lambda_{n} \in \rho_{1} \cap \rho_{2}$ for all $n$ with the property that $\lambda_{n}$ converges to $\lambda$. By Proposition $3.4 \mu^{2} / \lambda \in \sigma(S T)$ whenever $\mu \in \rho(S T) \cap$ $\rho(T S)$. In particular $\left(\lambda_{n}\right)^{2} / \lambda \in \sigma(S T)$ for all $n$. This is clearly impossible since $\left(\lambda_{n}\right)^{2} / \lambda$ converges to $\lambda$ and eventually $\left(\lambda_{n}\right)^{2} / \lambda \in N$.

The next two propositions give sufficient conditions for the hypothesis of Theorem 3.4 to be fulfilled. 
Proposition 3.6. If T, S, TS, and ST are closed and such that there exists a neighborhood of zero intersected with an open half plane about the origin which is a subset of $\rho(S T) \cap \rho(T S)$ then the state of $T S-\lambda I$ is the same as the state of $S T-\lambda I$ whenever $\mu \neq 0$.

Proof. Suppose $D=\{\mu|| \mu \mid<r\} \subset U$ is contained in $\rho(S T) \cap$ $\rho(T S)$, where $U$ denotes the open upper half plane.

Given $\lambda \neq 0$, choose $\alpha$ satisfying

(i) $0<|\alpha|<\min \{r,|\lambda|\}$;

(ii) argument of $\alpha$, $\arg \alpha$, is as follows:

(a) $\pi / 4$ if $\arg \lambda=0$

(b) $\arg \lambda$ if $0<\arg \lambda<\pi$;

(c) $3 \pi / 4$ if $\arg \lambda=\pi$;

(d) $\pi / 2+\arg \lambda / 4$ if $\pi<\arg \lambda<2 \pi$.

By direct calculation, it can be shown that both $\alpha$ and $\alpha^{2} / \lambda$ belong to $D \subset \rho(S T) \cap \rho(T S)$ and consequently by Theorem 3.4, the states of $T S-\lambda I$ and $S T-\lambda I$ agree. It is clear, by the method in which $\alpha$ was chosen, that our assumption of $U$ being the open upper half plane involves no loss of generality. Any other open half plane about the origin would simply introduce a change in $\arg \alpha$.

Note that if $S, T, S T$, and $T S$ are closed operators in a Hilbert space with both $S T$ and TS self-adjoint, the hypotheses of Proposition 3.6 hold.

Proposition 3.7. Let T, S, TS, and ST be closed and such that there exists a half plane entirely contained in $\rho(S T) \cap \rho(T S)$. Then the state of $S T-\lambda I$ is the same as the state of TS - $\lambda I$ whenever $\lambda \neq 0$.

Proof. Suppose that $U$ is a half plane contained in $\rho(S T) \cap \rho(T S)$. We may assume, without loss of generality, that

$$
U=\{\mu \mid I M(\mu)>R\} \text { where } R>1 .
$$

For $\lambda \neq 0$ we choose $\alpha$ as follows:

(i) If $\arg \lambda=0$, then $\arg \alpha=\pi / 4$ and $|\alpha|=\max \{\alpha R,|\lambda|\}$;

(ii) If $\arg \lambda=\pi$, then $\arg \alpha=3 \pi / 4$ and $|\alpha|=\max \{\alpha R,|\lambda|\}$;

(iii) If $0<\arg \lambda<\pi$, then $\arg \alpha=\arg \lambda$ and

$$
|\alpha|=\max \left\{|\lambda|, \frac{\alpha R}{\sin (\arg \lambda)}\right\}
$$

(iv) If $\pi<\arg \lambda<2 \pi$, then $\arg \alpha=\arg \lambda-\pi$ and

$$
|\alpha|=\max \left\{|\lambda|, \frac{R}{\sin (\arg \lambda-\pi)}\right\} \text {. }
$$


It can be demonstrated in a straight forward manner that both $\alpha$ and $\alpha^{2} / \lambda$ are in $\rho(S T) \cap \rho(T S)$ in each case.

4. Spectral decompositions. The notation in the following discussion is full explained in [5].

Theorem 4.1. If $D$ is a bounded Cauchy domain satisfying

$$
\partial D \subset \rho(S T) \cap \rho(T S)
$$

then there exists a pair of closed subspaces $\left(X_{1}, X_{2}\right)$ of $X$ and $\left(Y_{1}, Y_{2}\right)$ of $Y$ such that

(i) $\left(X_{1}, X_{2}\right)$ completely reduces $S T$;

(ii) $\left(Y_{1}, Y_{2}\right)$ completely reduces $T S$;

(iii) $(S T)_{1}=S T \mid X_{1}$ and $(T S)_{1}=T S \mid Y_{1}$ are continuous with domains $X_{1}, Y_{1}$ respectively;

(iv) $T: X_{i} \rightarrow Y_{i}, S: Y_{i} \rightarrow X_{i}, i=1,2$.

Proof. Let

$$
\begin{aligned}
& \sigma_{1}=D \cap \sigma_{e}(S T), \\
& \sigma_{2}=D \cap \sigma_{e}(T S),
\end{aligned}
$$

where $\sigma_{e}$ denotes the extended spectrum of the transformation. $\sigma_{1}$ and $\sigma_{2}$ are bounded spectral sets for $S T$ and $T S$ respectively. Let $\tau_{1}=\sigma_{e}(S T)-\sigma_{1}$ and $\tau_{2}=\sigma_{e}(T S)-\sigma_{2}$ be their complementary spectral sets.

If $E\left(\sigma_{1}\right), E\left(\sigma_{2}\right), E\left(\tau_{1}\right)$, and $E\left(\tau_{2}\right)$ are the projections associated with these spectral sets with ranges $X_{1}, Y_{1}, X_{2}$, and $Y_{2}$ respectively, it is well-known, see [5], that statements (i), (ii), and (iii) are satisfied.

For $x \in X, E\left(\sigma_{1}\right) x \in X_{1}$ and

$$
\begin{aligned}
T E\left(\sigma_{1}\right) x & =T\left[-\frac{1}{2 \pi i} \int_{+\partial D}(S T-\lambda I)^{-1} d \lambda\right] x \\
& =\left[-\frac{1}{2 \pi i} \int_{+\partial D} T(S T-\lambda I)^{-1} d \lambda\right] x \\
& =\left[-\frac{1}{2 \pi i} \int_{+\partial D}(T S-\lambda I)^{-1} d \lambda\right] T x \\
& =E\left(\sigma_{2}\right) T x .
\end{aligned}
$$

so $T: X_{1} \rightarrow Y_{1}$.

Similarly, if $x \in X, E\left(\tau_{1}\right) x \in X_{2}$ and

$$
\begin{aligned}
T E\left(\tau_{1}\right) & =T\left(I-E\left(\sigma_{1}\right)\right) x=T x-T E\left(\sigma_{1}\right) x \\
& =\left(I-E\left(\sigma_{2}\right)\right) T x=E\left(\tau_{2}\right) T x .
\end{aligned}
$$


So $T: X_{2} \rightarrow Y_{2}$.

In a similar manner $S: Y_{1} \rightarrow X_{1}, S: Y_{2} \rightarrow X_{2}$ which completes the proof of the theorem.

Theorem 4.2. If $D$ is a bounded Cauchy domain with

$$
\partial D \subset \rho(S T) \cap \rho(T S)
$$

and

$$
\sigma_{1}=D \cap \sigma_{e}(S T), \quad \sigma_{2}=D \cap \sigma_{e}(T S),
$$

then

$$
\left(\sigma_{1}-\sigma_{2}\right) \cup\left(\sigma_{2}-\sigma_{1}\right) \subset\{0\} .
$$

If in addition $0 \in D$, then

(i) the complementary spectral sets $\tau_{1}$ and $\tau_{2}$ are equal;

(ii) the state of $S T-\lambda I$ is the same as the state of $T S-\lambda I$, whenever $\lambda \neq 0$.

Proof. Using the notation of Theorem 4.1, let $T_{\imath}=T \mid X_{i}$, $S_{i}=S \mid Y_{i}, i=1,2$. Since $T_{i}, S_{i}, T_{i} S_{i}$, and $S_{i} T_{i}, i=1,2$, are restrictions of closed operators to closed subspaces, they are closed. Furthermore, $S_{i} T_{i}=(S T)_{i}, T_{i} S_{i}=(T S)_{i}$ for $i=1,2$.

By Theorem 4.1, $S_{1} T_{1} \in\left[X_{1}, X_{1}\right]$ and $T_{1} S_{1} \in\left[Y_{1}, Y_{1}\right]$ and therefore satisfy the hypotheses of Theorem 1.1. Thus for $\lambda \neq 0$, the state of $S_{1} T_{1}-\lambda E\left(\sigma_{1}\right)$ agrees with the state of $T_{1} S_{1}-\lambda E\left(\sigma_{2}\right)$.

When $0 \in D$ the sets $\sigma\left(S_{2} T_{2}\right)=\tau_{1}$ and $\sigma\left(T_{2} S_{2}\right)=\tau_{2}$ are bounded away from zero. Consequently by Proposition 3.6, the state of $S_{2} T_{2}$ $\lambda E\left(\tau_{1}\right)$ agrees with the state of $T_{2} S_{2}-\lambda E\left(\tau_{2}\right)$ whenever $\lambda \neq 0$.

It can be seen that the above is both necessary and sufficient for the state of $S T-\lambda I$ to be the same as the state of $T S-\lambda I$.

From the preceding theorems we obtain the final results:

Theorem 4.3. Suppose $0 € \rho(T S) \cap \sigma(S T)$ and a bounded Cauchy domain $D$ exists satisfying:

(i ) $\partial D \subset \rho(S T) \cap \rho(T S)$;

(ii) $0 \in D$.

If $\sigma_{1}, \cdots, \sigma_{n}$ is a spectral decomposition of $\sigma_{e}(S T)$ then $\sigma_{0}, \sigma_{1}$, $\cdots, \sigma_{n}$ is a spectral decomposition of $\sigma_{e}(T S)$ where

$$
\sigma_{0}=\{0\}
$$

whenever $0 \in \sigma(T S) \cap \rho(S T)$, and is empty otherwise.

Moreover, if $E_{i}(S T)$ and $E_{i}(T S)$ are the projections associated with these spectral sets with ranges $X_{i}$ and $Y_{i}$ respectively, then 


$$
\begin{aligned}
& T: X_{i} \longrightarrow Y_{i} ; \\
& S: Y_{i} \longrightarrow X_{i}
\end{aligned}
$$

where $i=1, \cdots, n$, and when $0 \in \sigma(T S) \cap \rho(S T)$,

$$
S: Y_{0} \longrightarrow\{0\} \text {. }
$$

Proof. First note that by Theorem 4.2,

$$
\left(\sigma_{e}(S T)-\sigma_{e}(T S)\right) \cup\left(\sigma_{e}(T S)-\sigma_{e}(S T)\right) \subset\{0\}
$$

and since $\sigma_{e}(S T)$ and $\sigma_{e}(T S)$ are both closed subsets of the complex plane, if $0 \in \sigma(T S) \cap \rho(S T)$ it must be an isolated point in $\sigma(T S)$. This demonstrates that the spectral decomposition $\sigma_{1}, \cdots, \sigma_{n}$ of $\sigma_{e}(S T)$ gives rise to the spectral decomposition $\sigma_{0}, \sigma_{1}, \cdots, \sigma_{n}$ of $\sigma_{e}(T S)$.

If $\infty \in \sigma_{e}(S T)$, i.e., if $S T \notin[X, X]$, assume that $\infty \in \sigma_{n}$. Then $\sigma_{1}, \cdots, \sigma_{n-1}$ are bounded spectral sets for both $S T$ and $T S$.

Let $D_{i}$ be an admissible domain for $\sigma_{i}, i=1, \cdots, n-1$. Then

and

$$
E_{i}(S T)=-\frac{1}{2 \pi i} \int_{+\partial D_{i}}(S T-\lambda I)^{-1} d \lambda
$$

$$
E_{i}(T S)=-\frac{1}{2 \pi i} \int_{+\partial D_{i}}(T S-\lambda I)^{-1} d \lambda .
$$

By Theorem 4.1,

$$
\begin{aligned}
& T: X_{i} \longrightarrow Y_{\imath}, \\
& S: Y_{\imath} \longrightarrow X_{i},
\end{aligned}
$$

$i=1, \cdots, n-1$, moreover $T, S$ are continuous and everywhere defined on these subspaces.

Further, if $0 \in \sigma(T S) \cap \rho(S T)$ and $D_{0}$ is an admissible domain for $\sigma_{0}$, let $y \in Y_{0}$. By Theorem 4.1, $y \in D(S)$ and

$$
\begin{aligned}
S y & =S E_{0}(T S) y \\
& =S\left(-\frac{1}{2 \pi i} \int_{+\alpha D_{0}}(T S-\lambda I)^{-1} d \lambda\right) y \\
& =\left(-\frac{1}{2 \pi i} \int_{+\alpha D_{0}}(S T-\lambda I)^{-1} d \lambda\right) S y \\
& =0 .
\end{aligned}
$$

To show that $T: X_{n} \rightarrow Y_{n}, S: Y_{n} \rightarrow X_{n}$ observe that

$$
E_{n}(S T)=I-\sum_{i=1}^{n-1} E_{i}(S T)
$$




$$
E_{n}(T S)=I-\sum_{i=0}^{n-1} E_{\imath}(T S)
$$

where $E_{0}(T S)=0$ if $0 \in \rho(T S)$.

When $T \in[X, Y], S \in[Y, X]$ we clearly have a bounded Cauchy domain

$$
D=\{\mu|| \mu \mid<\max (\|S T\|,\|T S\|)+1\}
$$

which satisfies the conditions of Theorem 4.3. Hence:

CoRollary 4.1. If $T \in[X, Y], S \in[Y, X]$ and $0 \notin \rho(T S) \cap \sigma(S T)$ then a spectral decomposition $\sigma_{1}, \cdots, \sigma_{n}$ of $\sigma(S T)$ gives a spectral decomposition $\sigma_{0}, \sigma_{1}, \cdots, \sigma_{n}$ of $\sigma(T S)$ where

$$
\sigma_{0}=\left\{\begin{array}{cl}
\{0\} & \text { whenever } 0 \in \sigma(T S) \cap \rho(S T) \\
\dot{\phi} & \text { otherwise }
\end{array}\right.
$$

Moreover, if $E_{i}(S T)$ and $E_{i}(T S)$ are the projections associated with the spectral sets with ranges $X_{i}$ and $Y_{i}$ respectively, then $T: X_{i} \rightarrow Y_{i}, \quad S: Y_{i} \rightarrow X_{i}, \quad i=1, \cdots, n$ and when $0 \in \sigma(T S) \cap \rho(S T)$, $S: Y_{0} \rightarrow\{0\}$.

\section{REFERENCES}

1. S. Banach, Théorie des Opérations Linéares, Monografje Matematyczne, Warsaw, 1932.

2. S. K. Berberian, Approximate proper vectors, Proc. Amer. Math. Soc., 13 (1962), 111-114.

3. N. Dunford and J. T. Schwartz, Linear Operators, Part I, Interscience Publishers, Inc., New York, 1958.

4. S. Goldberg, Unbounded Linear Operators, McGraw-Hill, New York, 1966.

5. A. E. Taylor, Functional Analysis, Wiley, New York, 1958.

6. - Spectral theory of closed distributive operators, Acta. Math., 84 (1950), $189-224$.

Received Mach 16, 1973 and in revised form September 21, 1973. The results in this paper were part of the author's Ph.D. thesis directed by Professor Sidney Birnbaum at the University of South Carolina.

Youngstown State University 



\section{PACIFIC JOURNAL OF MATHEMATICS}

\section{EDITORS}

RICHARD ARENS (Managing Editor)

University of California

Los Angeles, California 90024

R. A. Beaumont

University of Washington

Seattle, Washington 98105
J. DugundJI

Department of Mathematics University of Southern California Los Angeles, California 90007

D. Gilbarg AND J. Milgram Stanford University

Stanford, California 94305

\section{ASSOCIATE EDITORS}
E. F. BECKENBACH
B. H. NeUmanN
F. WOLF
K. YOSHIDA

\section{SUPPORTING INSTITUTIONS}

UNIVERSITY OF BRITISH COLUMBIA CALIFORNIA INSTITUTE OF TECHNOLOGY

UNIVERSITY OF CALIFORNIA

MONTANA STATE UNIVERSITY

UNIVERSITY OF NEVADA

NEW MEXICO STATE UNIVERSITY

OREGON STATE UNIVERSITY

UNIVERSITY OF OREGON

OSAKA UNIVERSITY
UNIVERSITY OF SOUTHERN CALIFOF

STANFORD UNIVERSITY

UNIVERSITY OF TOKYO

UNIVERSITY OF UTAH

WASHINGTON STATE UNIVERSITY UNIVERSITY OF WASHINGTON

AMERICAN MATHEMATICAL SOCIET NAVAL WEAPONS CENTER 


\section{Pacific Journal of Mathematics}

\section{Vol. 54, No. 1 \\ May, 1974}

Ralph K Amayo, Engel Lie rings with chain conditions ..................

Bernd Anger and Jörn Lembcke, Hahn-Banach type theorems for hypolinear

functionals on preordered topological vector spaces ..................

Gregory Frank Bachelis and Samuel Ebenstein, On $\Lambda(p)$ sets ................

Harvey Isaac Blau, Indecomposable modules for direct products of finite

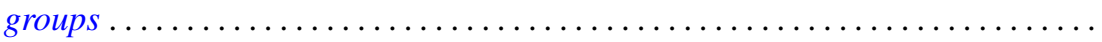

Larry Eugene Bobisud and James Calvert, Singular perturbation of a

time-dependent Cauchy problem in a Hilbert space ................

Walter D. Burgess and Robert Raphael, Abian's order relation and orthogonal

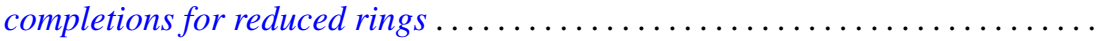

James Diederich, Representation of superharmonic functions mean continuous at

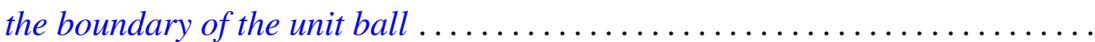

Aad Dijksma and Hendrik S. V. de Snoo, Self-adjoint extensions of symmetric

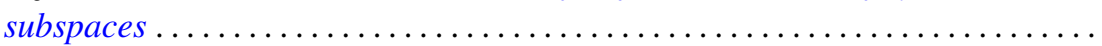

Gustave Adam Efroymson, A Nullstellensatz for Nash rings . . . . . . . . . . . . .

John D. Elwin and Donald R. Short, Branched immersions onto compact orientable surfaces . . . . . . . . . . . . . . . . . . . . . . . . .

John Douglas Faires, Comparison of the states of closed linear

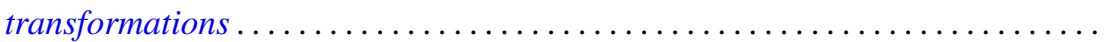

Joe Wayne Fisher and Robert L. Snider, On the von Neumann regularity of rings with regular prime factor rings .

Franklin Takashi Iha, A unified approach to boundary value problems on compact intervals

Palaniappan L. Kannappan and Che Tat $\mathrm{Ng}$, On functional equations connected with directed divergence, inaccuracy and generalized directed divergence

Samir A. Khabbaz and Elias Hanna Toubassi, The module structure of Ext $(F, T)$ over the endomorphism ring of $T$...

Garo K. Kiremidjian, On deformations of complex compact manifolds with boundary.

Dimitri Koutroufiotis, Mappings by parallel normals preserving principal

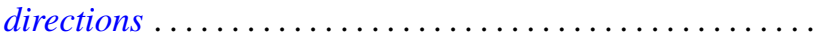

W. K. Nicholson, Semiperfect rings with abelian adjoint group

Norman R. Reilly, Extension of congruences and homomorphisms to translational hulls

Sadahiro Saeki, Symmetric maximal ideals in $M(G)$

Brian Kirkwood Schmidt, On the homotopy invariance of certain functors ...

H. J. Shyr and T. M. Viswanathan, On the radicals of lattice-ordered rings ...

Indranand Sinha, Certain representations of infinite group algebras ...

David Smallen, The group of self-equivalences of certain complexes ...

Kalathoor Varadarajan, On a certain problem of realization in homotopy

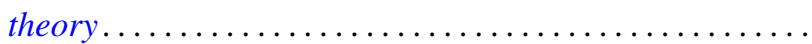

